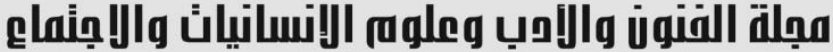

Journal of Arts, Literature, Humanities and Social Sciences www.jalhss.com

\section{الضيق الجندري}

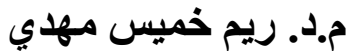

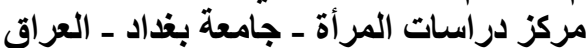
reem.m@wsc.uobaghdad.edu.iq الايميل

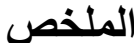

يُعد مصطلح الضيق الجندري من الموضو عات التي تحمل بُعداً نفسياو اجتماعياو طبيا في آنٍ واحدٍ ، و هذا ما

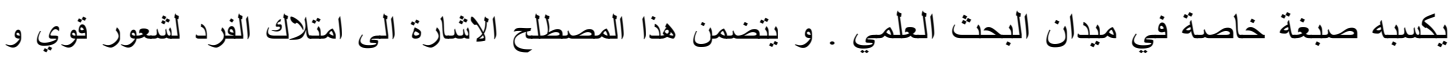

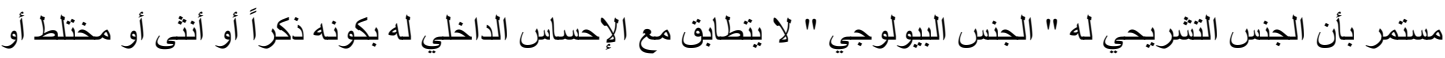

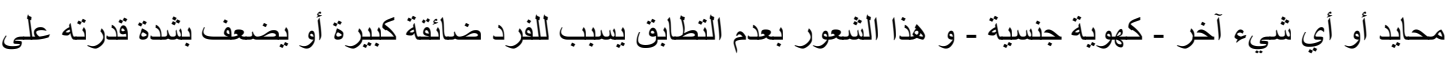
العمل و الاندماج المجتمعي ـ و لا يصنف الضيق الجندري من ضمن الاضطر ابات الجنسية و لا العقلية كما أنشارت لذلك

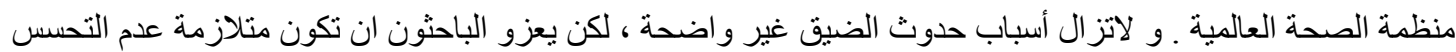
للاندروجين ، و تضخم الغدد الكظرية من الأسباب الكامنة وراء الاصابة به ـ برتبط الضيق الجندري بعدد من الأزمات النفسية و الاضطر ابات العقلية ، و تزداد نسب الانتحار بين الأفر اد المصابين بالضيق الجندري بالمقارنة مع عموم السكان. و في الختام ، تم التوصل الى ضرورة زيادة الوعي الأسري بالتغيرات الجندرية لأبنائهم و منذ الصغر و محاولة تسليط الضوء على الحالات الخاصة من القضايا الجندرية في وسائل الاعلام و تقليل حجم التوتر و العدائبة نحو هذه الفئة 


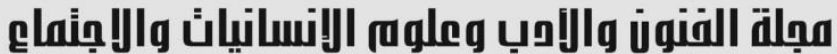 \\ Journal of Arts, Literature, Humanities and Social Sciences www.jalhss.com
}

\section{Gender Dysphoria}

\author{
Dr. Reem Kh. Mahdi \\ Women's Studies Center - University of Baghdad - Iraq \\ Email: reem.m@wsc.uobaghdad.edu.iq
}

\begin{abstract}
The term Gender Dysphoria is one of the subjects that have a psychological, social and medical dimensions at the same time, and this is gaining a special character in the field of scientific research. The term refers to an individual having a strong and persistent feeling that biological sex does not match his/ her inner sense of being male, female, mixed, neutral or anything else - as a sexual identity - and this sense of mismatch causes the individual in distress or severely impair his/ her ability to work and community integration .Gender Dysphoria is not classified as a sexual or mental disorder, as indicated by the World Health Organization .

The causes of the Gender Dysphoria are still not clear, but the researchers attribute the Androgen Insensitivity Syndrome and adrenal enlargement to be the underlying causes.

Gender Dysphoria is associated with a number of psychological problems and mental disorders. Suicide rates among individuals with Gender Dysphoria are higher than in the general population .

Conclusion : It is a necessity of increasing family awareness of the gender transition of their children and since childhood, and trying to shed light on the special cases of gender issues in the media and reduce the amount of tension and hostility towards this group .
\end{abstract}

Keywords: Dysphoria, Gender, Gender Dysphoria . 


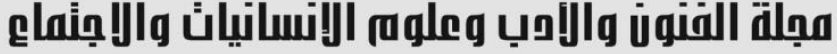

Journal of Arts, Literature, Humanities and Social Sciences www.jalhss.com

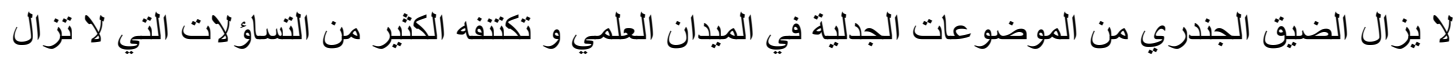

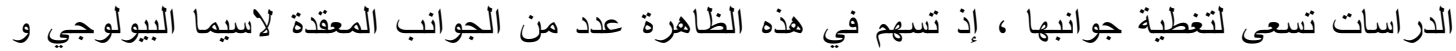

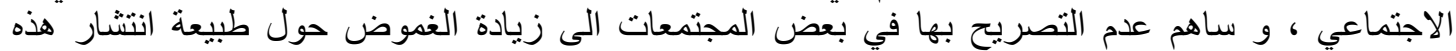
الظاهرة و أثرها على الفرد و البيئة التي ينتمي اليها بالاضافة الى زيادة معاناة هذه الثريحة و في إمكانية

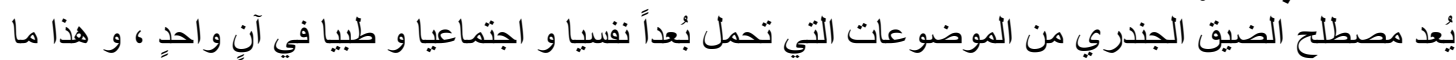

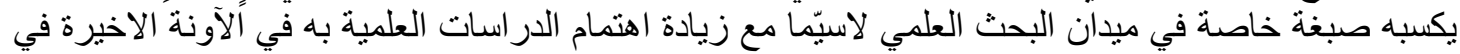

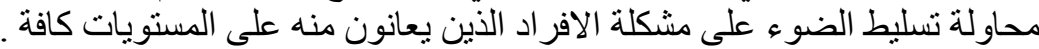

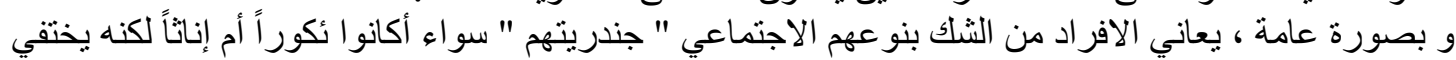
بمرور الوقت ، أما هذا المصطلح فيشير الى شعور بعدم القناعة بالنوع الاجتماعي للفرد لمدة طويلة من الزمن ، . ( CPA , 2014 )

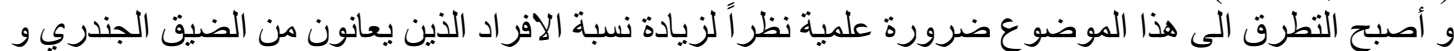

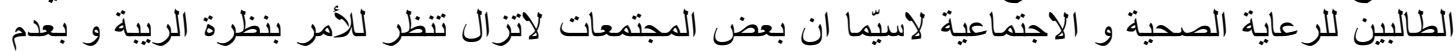

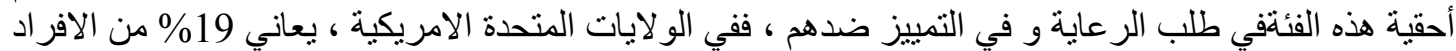

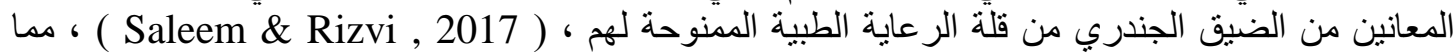

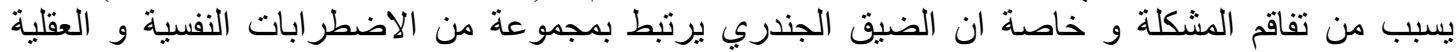

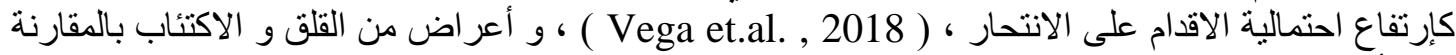

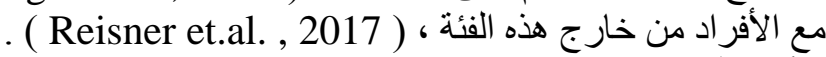

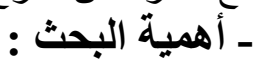
يشهد العصر الحالي إزدياد كبير في المو اضيع المتعلقة بالقضايا الجنسية و الجندرية و اهتمام الوعي الجماهيري

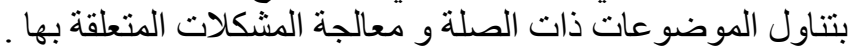

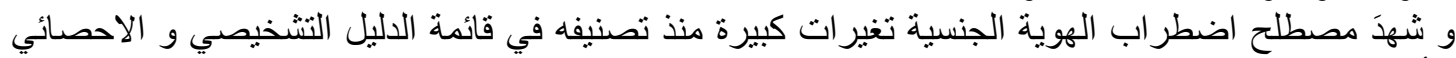

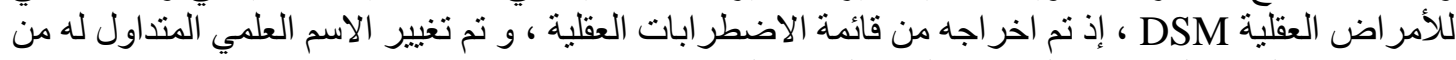

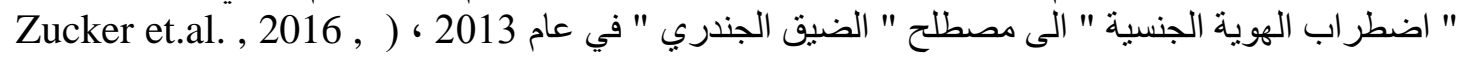
. ( p.20.1

و أثنارت الرابطة المهنية العالمية لصحة المتحولين جنسياً (WATh (WPATH ) Transgender Health

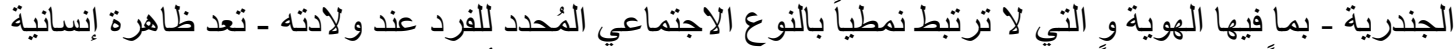

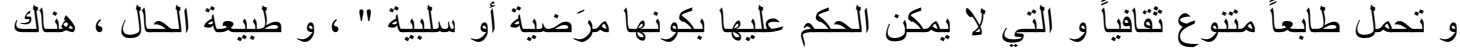

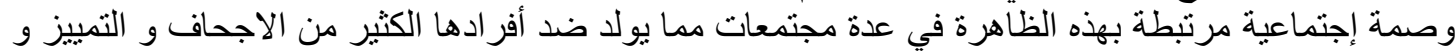

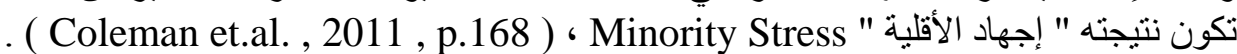

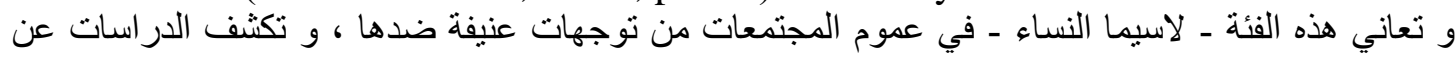

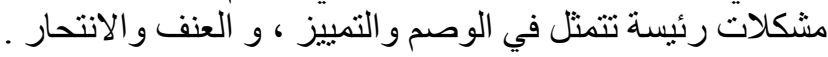

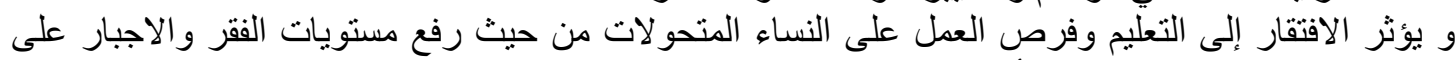

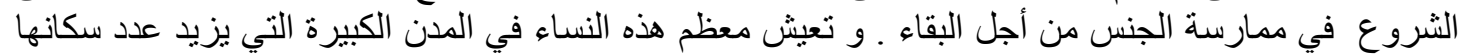

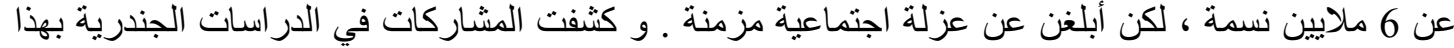

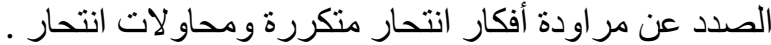
و تؤكد الدر اسات الاستقصائية الموجزة النتائج التي توصلت التئت إليها من حيث الانتشار الواسع للتمييز ، والانتحار ، و وانخفاض الدعم الاجتماعي ، ( de Jesus et.al. , 2020 ) . 


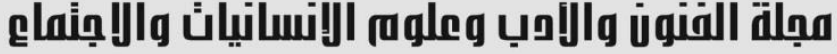

Journal of Arts, Literature, Humanities and Social Sciences www.jalhss.com

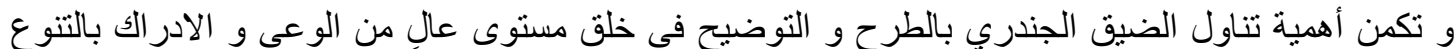

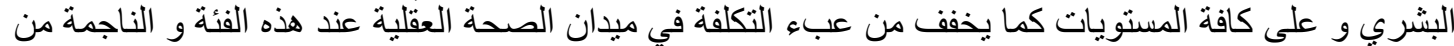

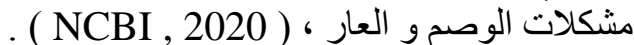

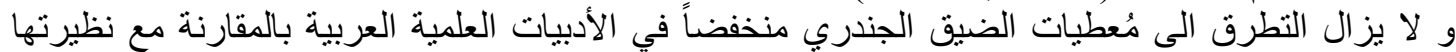

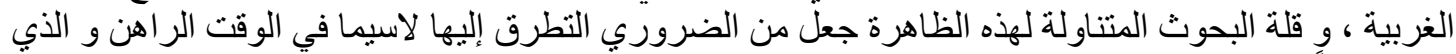

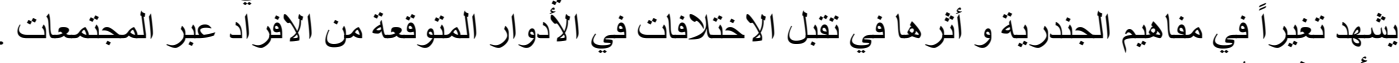

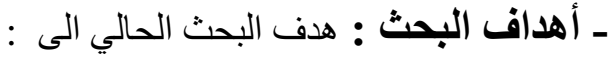

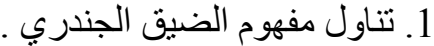

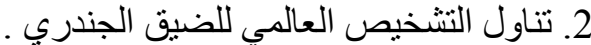

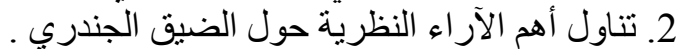
ـ حدود البحث :تحدد البحث الحالي بتناول الأدبيات و الدراسات العلميةو التي تناولت مصطلح الضيق

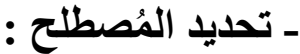
الضيق الجندري Gender Dysphoria

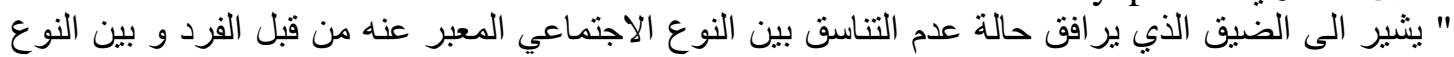

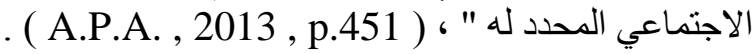

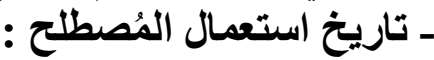
تم استعمال مُصطلح الضيق الجندري " اضطراب الهُبط الهوية الجنسية سابقاً "لأول مرة من قبل فيسك Fisk عام 1974 لوصف حالة الأفراد الذين يعانون من عدم الرغبة في طبيعة جنسهم الحالي و الرغبة فية بإعادة تشكيل

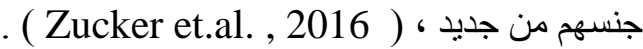

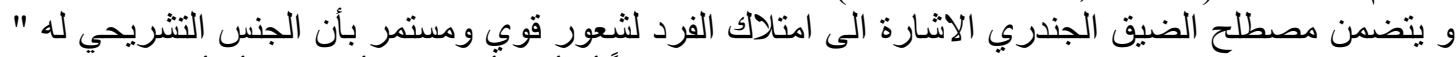

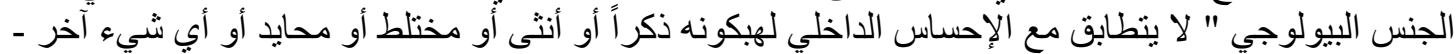

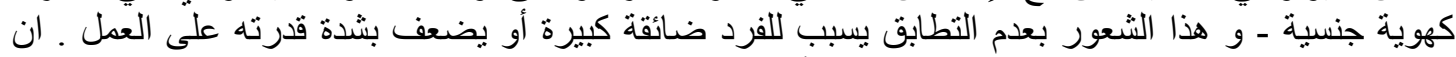

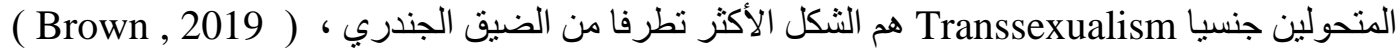

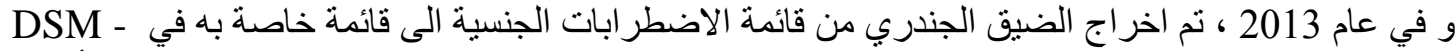
5، و فصل حالة الضيق عند الاطفال عن حالة الضيق عند البالغين تحت فئة " الضيق الجندئ الضدري في الأطفال قGender Dysphoria in Children"

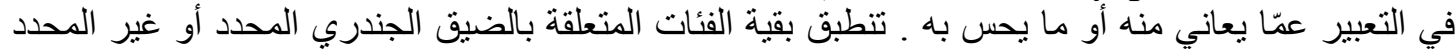
Specified or non specified يشعر بحالة الضيق من نوعه الاجتماعي ، ( A.P.A. , 2013 , P.451-460 ) . أما المتحولون اللون جنسياً

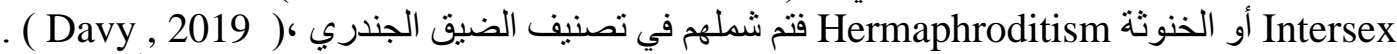
و حذفت منظمة الصحة العالمية حالة الضيق الجندري " اضطر اب الهوية الجنسية " من قائمة الأمر اض العقلية

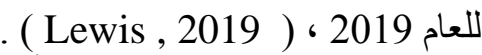

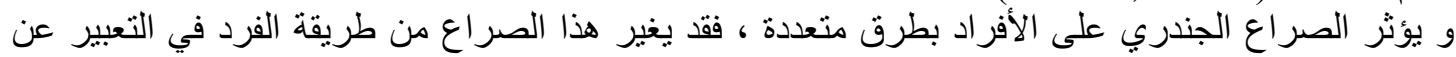

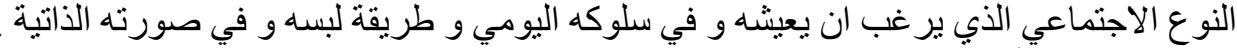

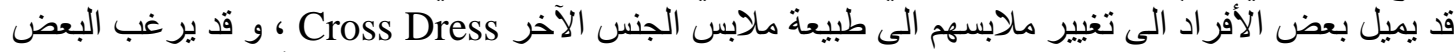

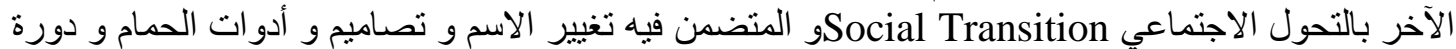

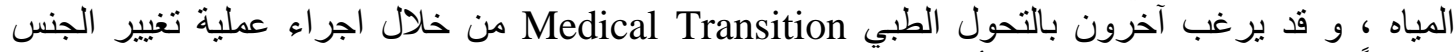

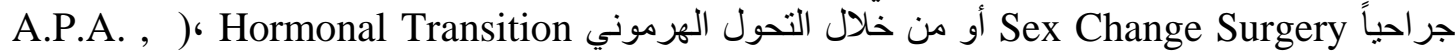




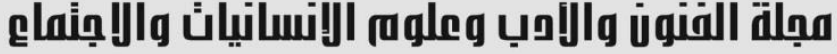

Journal of Arts, Literature, Humanities and Social Sciences www.jalhss.com

أ. الرغبة في ان يكون من الجنس الآخر أو الاصرار على أن يكون مثله ( أو أي نوع اجتماعي مغاير لها هو

ب. عند الصبية " النوع المحدد " ، هناك رغبة في خلع الملابس أو محاكاة ملابس الاناث ، و و عند الفتيات "

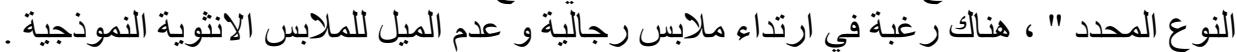

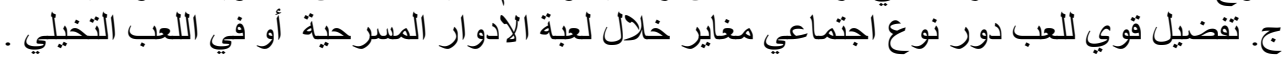

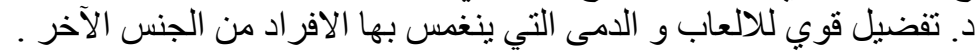

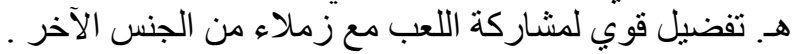

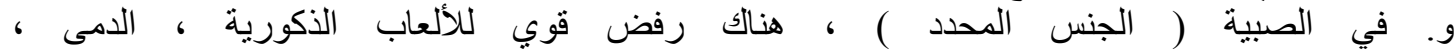
و الأنشطة ذات الطابع الذكوري و تجنب خشونة وتعثر اللعب ، و في الفتيات( الجنس المحدد ) ، هنالك رفض الأل

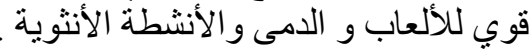

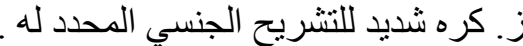

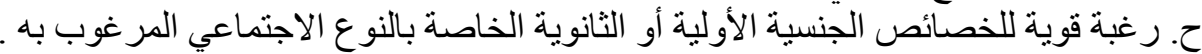

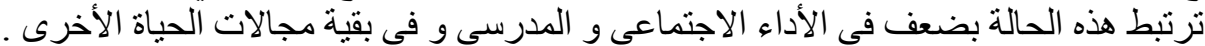

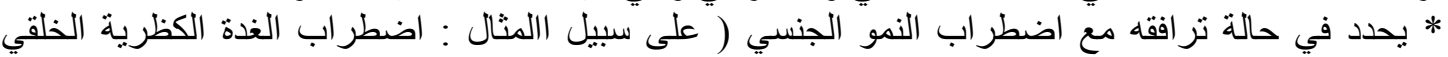

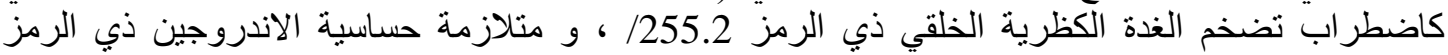

ـ الضيق الجندري عند المراهقين و البالغين : رمز

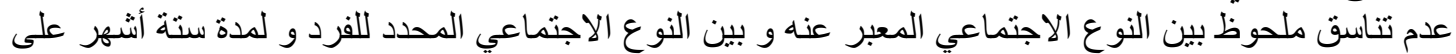
ان تانضمن اثتين من المعايير الآتية :

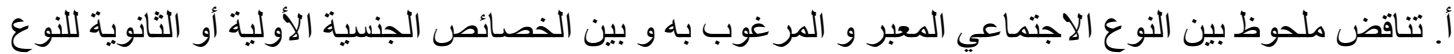

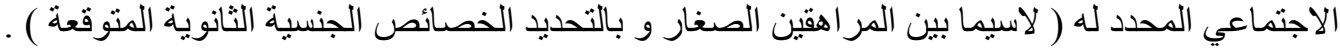

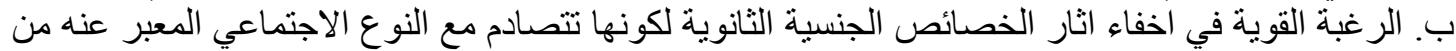

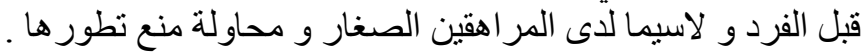

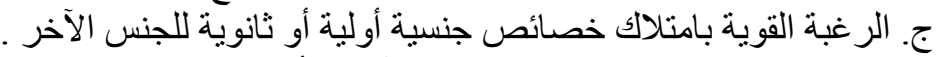

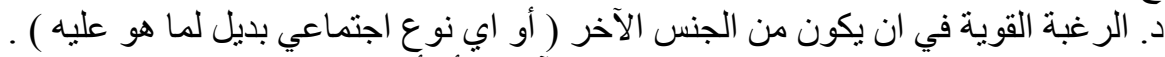

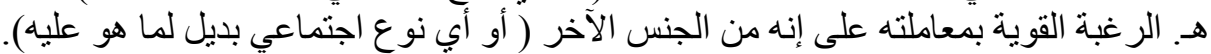

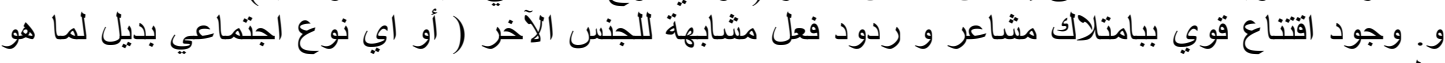
عليه). ترتبط هذه الحالة بضعف في الأداء الاجتماعي و المهني و في بقية مجالات الحباة الأخرى .

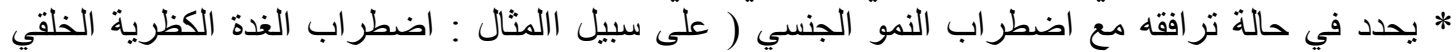

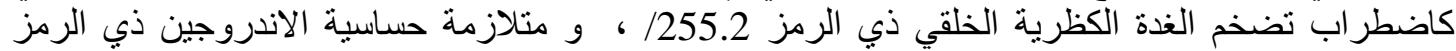
( 259.50

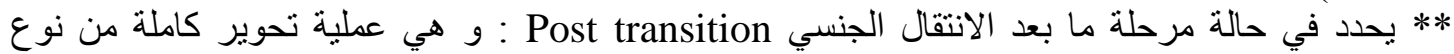

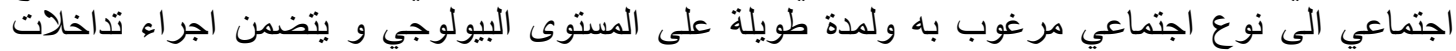

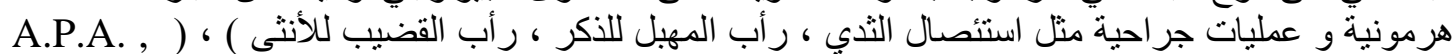
. (2013, pp. 452-453

و في تصنيف منظمة الصحة العالمية 10 ICD للاهوية الجندرية Gender Identity المرمز F64 للعام 2016 ،

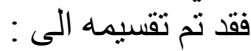

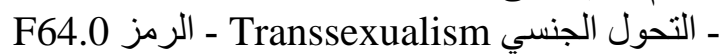

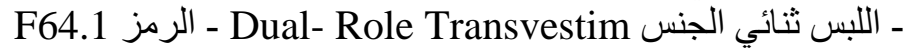
ـ اضطر اب الهوية الجنسية للطفولة Gender Identity Disorder of Childhood ـ الرمز ـ اضطر ابات هوية جنسية أخرى Other Gender Identity Disorders - الرمز

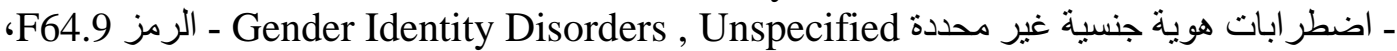




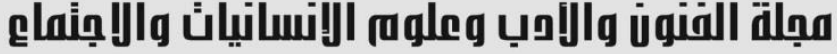

Journal of Arts, Literature, Humanities and Social Sciences www.jalhss.com

لا تزال أسباب حدوث الضيق الجندري غير واضحة حتى يومنا هذا ، لكن يعزو الباحثُون في هذا المجال الى الى الصي

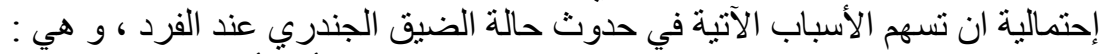

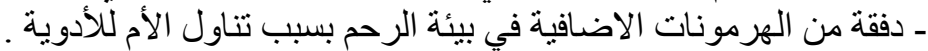

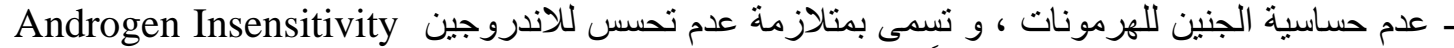
Syndrome AIS

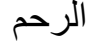

ـ تضخم الغدة الكظرية الخلقي - (CAH) حيث يتم إنتاج مستويات عالية من الهرمونات الذكرية في الجنين

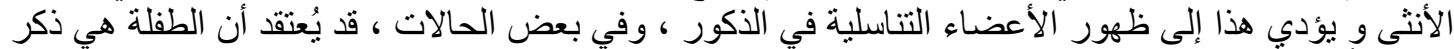

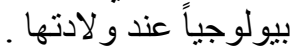

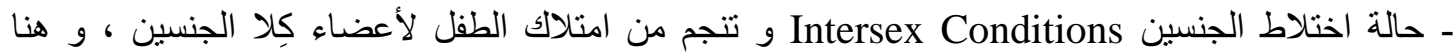

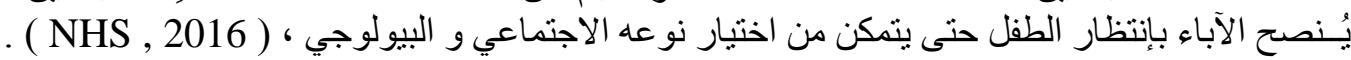

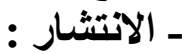

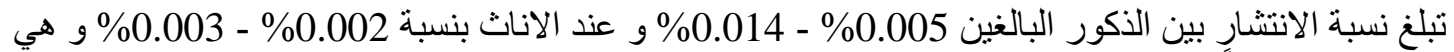

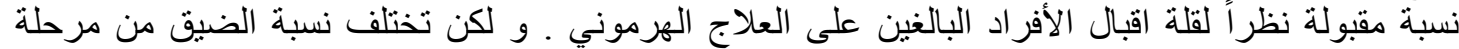

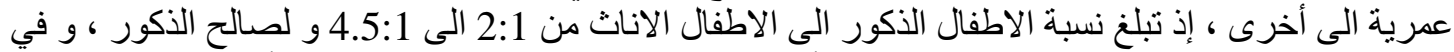

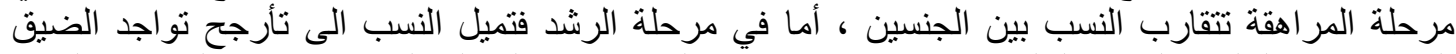

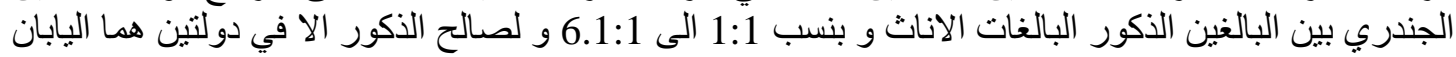

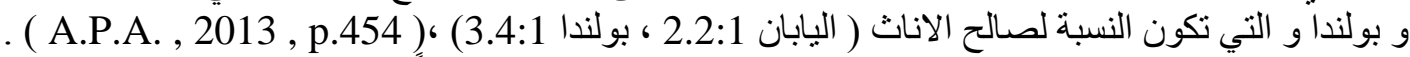

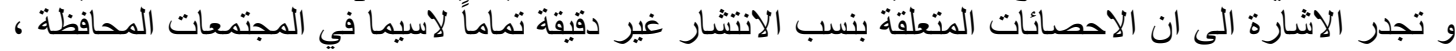

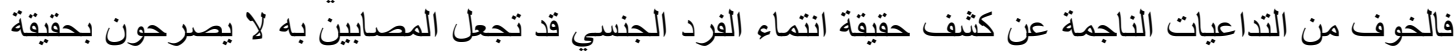

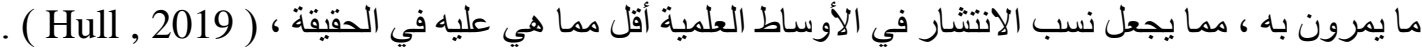

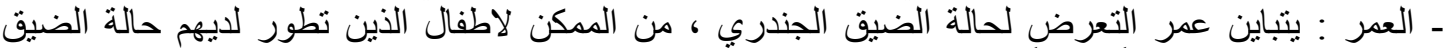

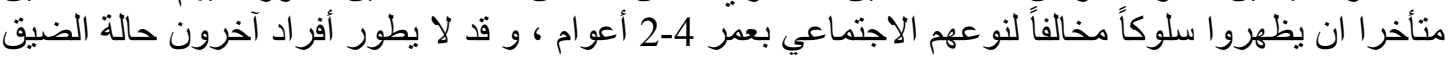

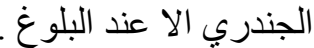
و عادةً ما يبدأ نمو الهوية الجنسبة بعمر 2-4 أعوام و بالنتيجة يعد حدوث الضيق الضيق الجندرية متوقعاً في هذه الفترة

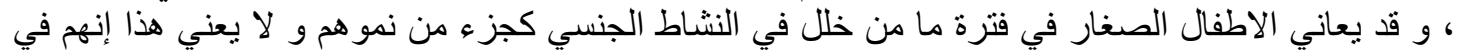

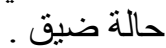
و من جُملة العو امل المساهمة في حالة الضيق الجندري :

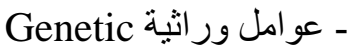

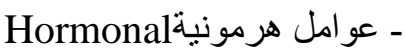

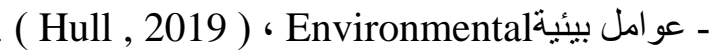

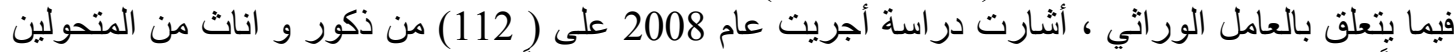

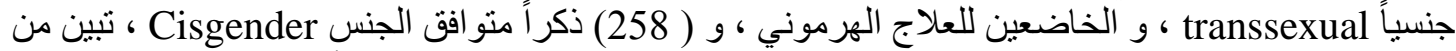

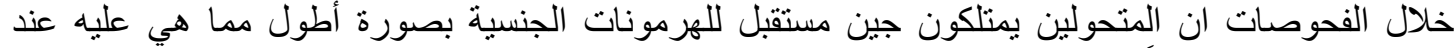

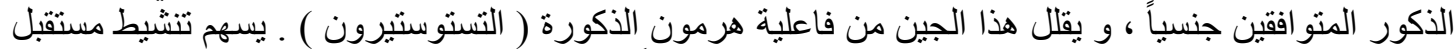

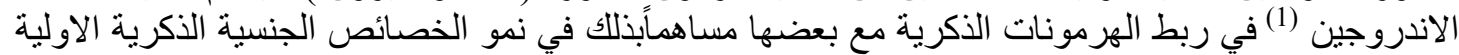

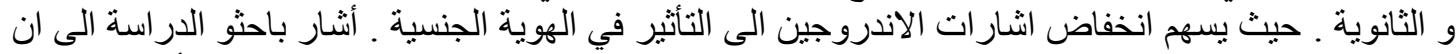

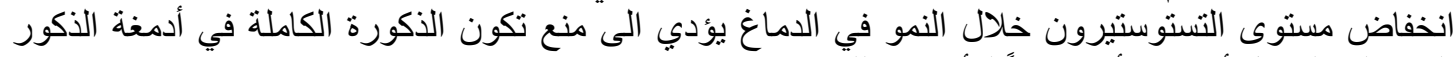

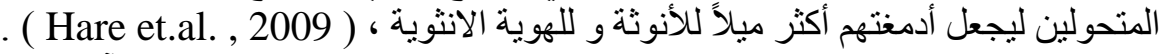

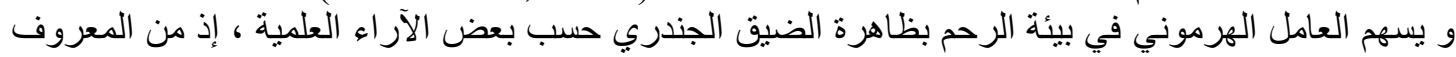

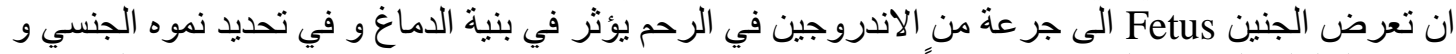

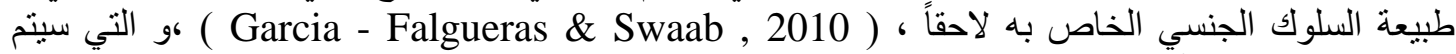
توضيحها في فقرة الآراء النظرية النية لاصنا 


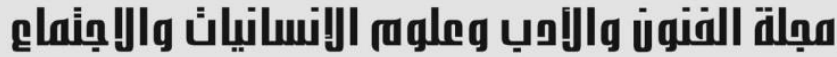 \\ Journal of Arts, Literature, Humanities and Social Sciences www.jalhss.com}

Volume (59) October 2020

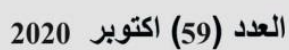

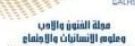

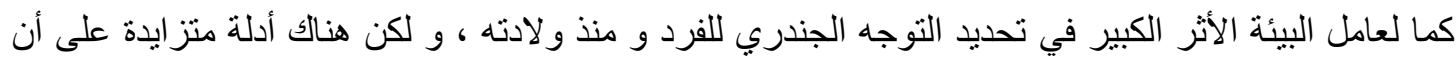

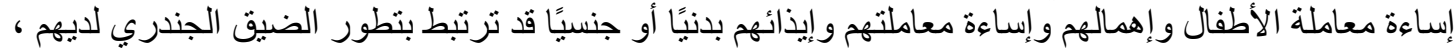

. ( Garg , 2020 )

ـ الآراء النظرية :

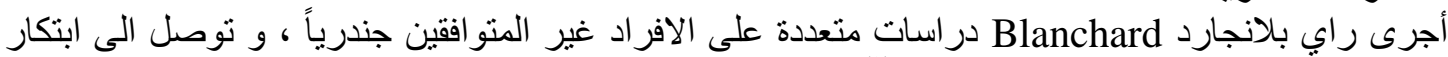

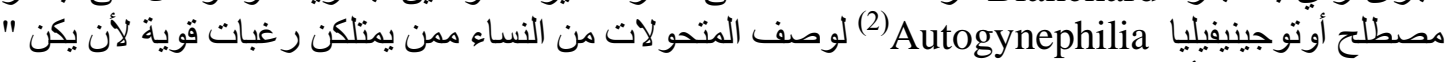
نساء " بالفعل ، و أنشار الى ان للضيق الجندري نوعين : ضيق جندري مثلي Homosexual و ضين لأنيق جندري

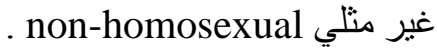
و قدّم الدصطلح أتوجينيفيليك Autogynephilic للاشارة الى نزعة رجل رجل في اثنارة جسده جنسياً من خلال فكرة

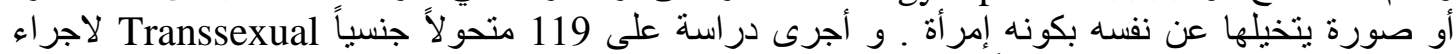

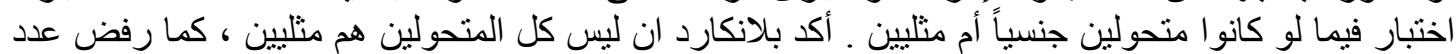

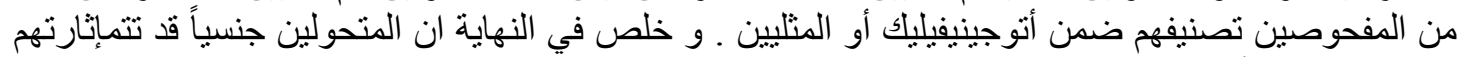

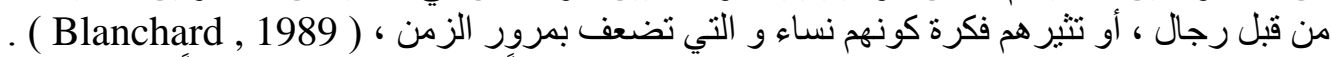

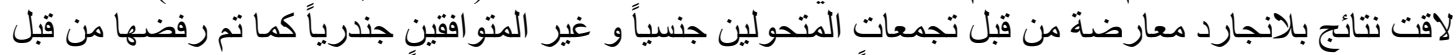

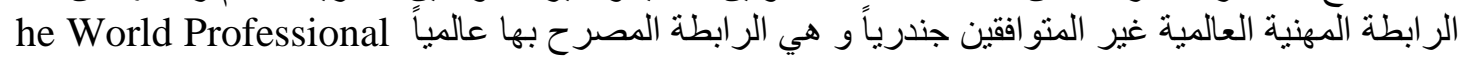
Krudson et.al. , ) ، لعدم كفاية الأدلة التجريبيا (Association for Transgender Health (WPATH

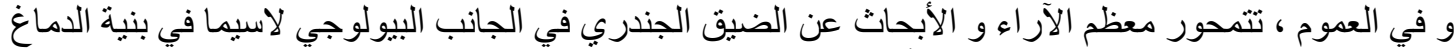

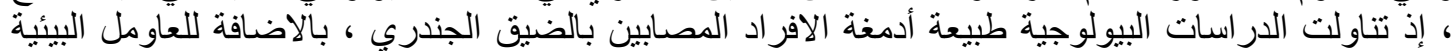

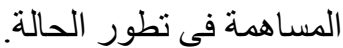

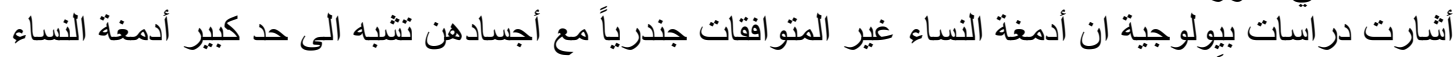

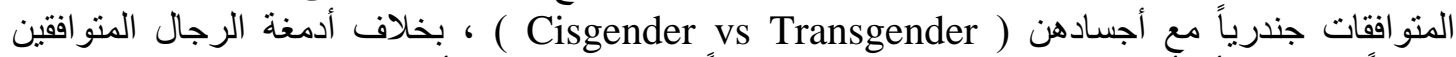

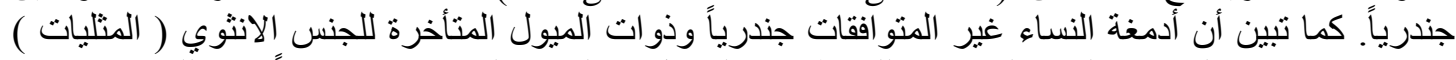
Gynephilia

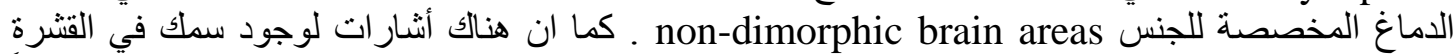
الدماغية Cortical Thickness عند المساء المتوافقات و غير المتو افقات لكن بأماكن مختلفة و هي أكثر سمكاً

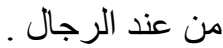
أما بالنسبة للرجال غير المنو افقين جندرياً و الميالون للجنس الذكري ( المثليين ) Androphilia ، فقد كان لديهم

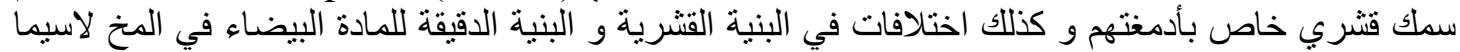
في النصف الأيمن منه ، ( Guillamon et.al. , 2016 ) .

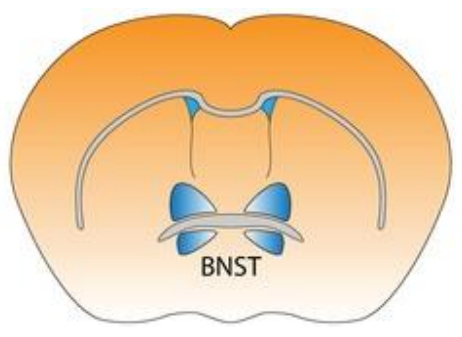

شكل (1) نواة السطر الطرفي ( الانتهائي ) عند الفئران ،

( Wikipedia ) 


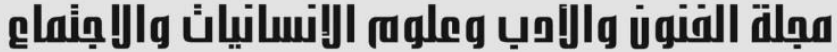 \\ Journal of Arts, Literature, Humanities and Social Sciences www.jalhss.com

و تثير دراسات حديثة بهذا الصدد الى احتمالية ربط ظاهرة الضيق الجندري بالعلوم العصبية Neuroscience

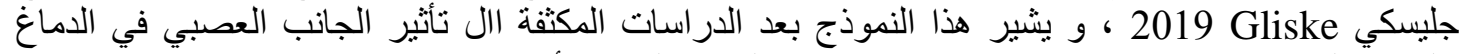

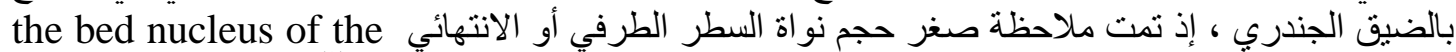
stria terminalis (BNST)

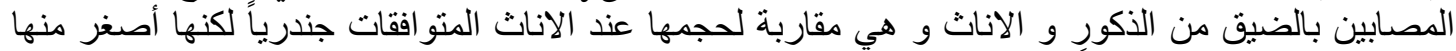

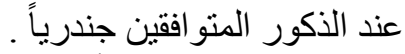

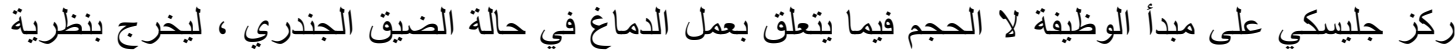

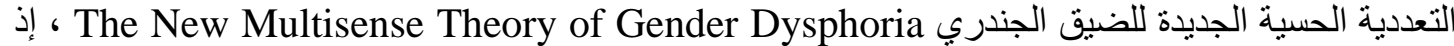
تسهم مجموعة عو امل مصيرية في إحداث الخلل في التنظيم الجندري للفرد .

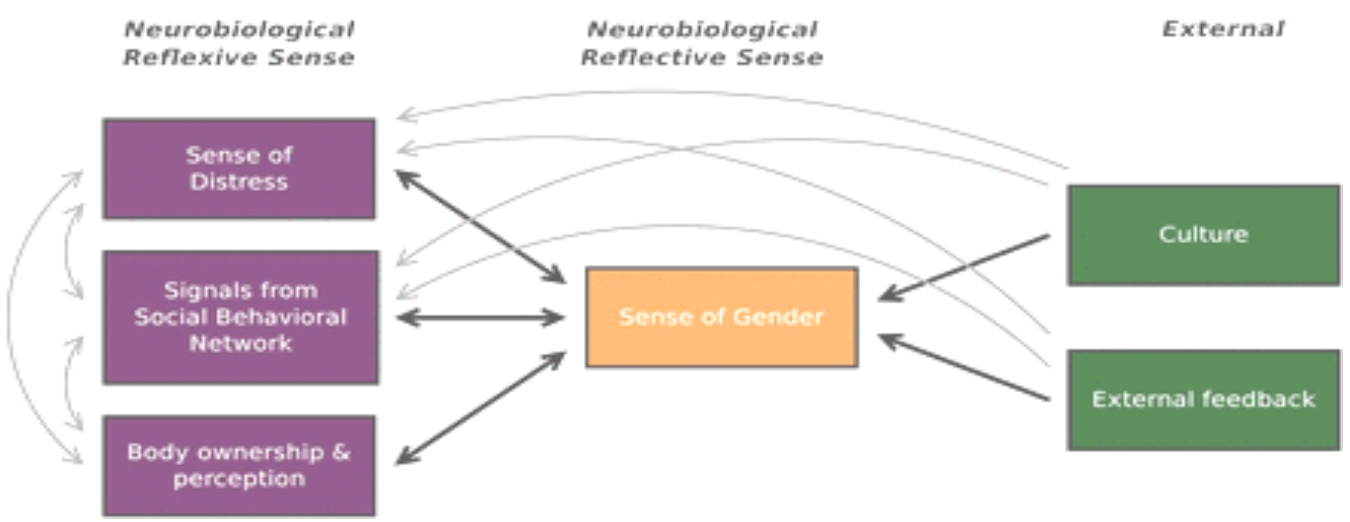

\section{شكل (2) رسم تخطيطي لنظرية التعددية الحسية لـ كليسكي}

\section{( Gliske , 2019 )}

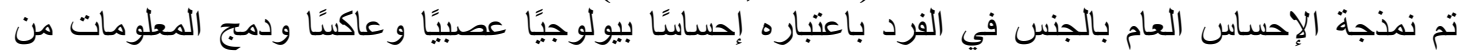

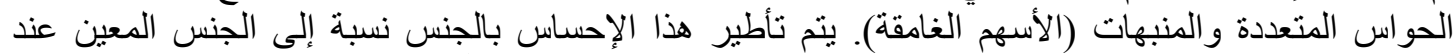

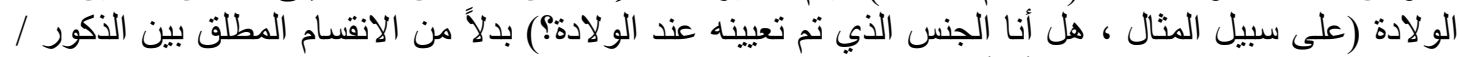

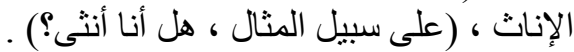

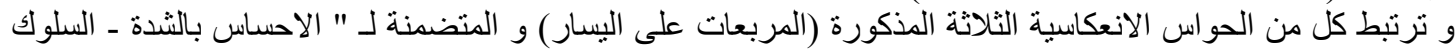

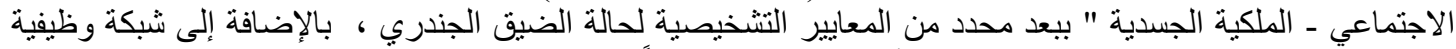

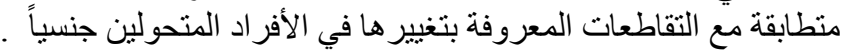

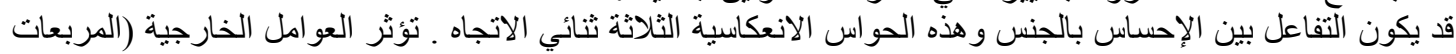

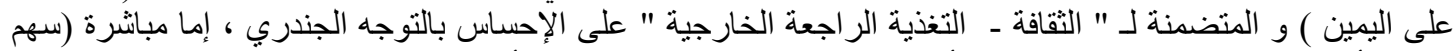

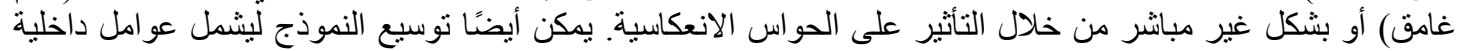

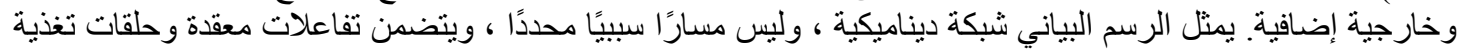

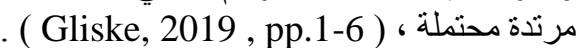

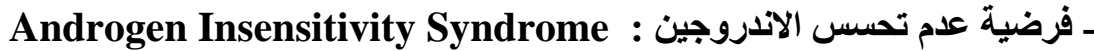
تحدث هذه المتلازمة بسبب خلل جيني ، و فيها يصبح الجسم البشري غير قادر على الاستجابة للاندروجينات ـو هذا الخلل

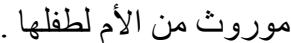
في بيئة الرحم ، يستقبل الجنين مجمو عة من الهرمونات لإكمال تحديد جنسه سواء أنثى أو ذكر ـ تمتلألك الأنثى من و الديها

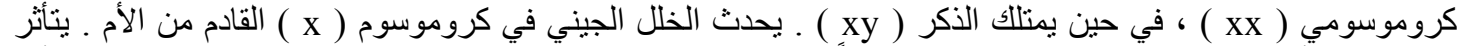

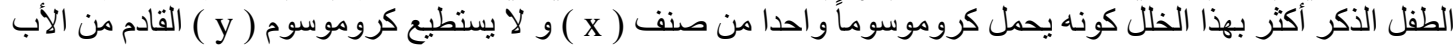

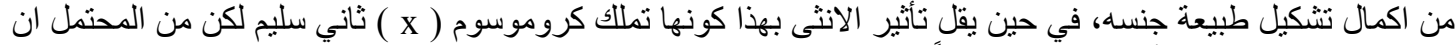




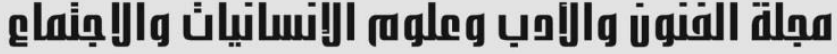

\author{
Journal of Arts, Literature, Humanities and Social Sciences
} www.jalhss.com

1. فرصة 4:1 أن تنجب أنثى غير متأثرة بالجين لكنها حامل له .

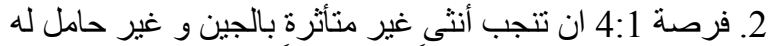

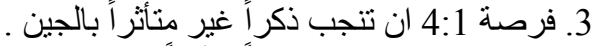

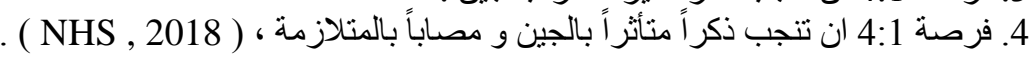
و اللمتلازمة صنفان : أنجان

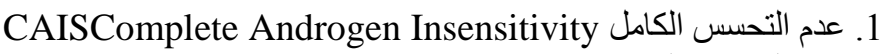
2. عدم التحسس الجزئي Partial Androgen Insensitivity PAIS

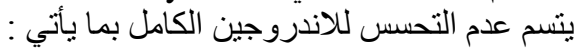

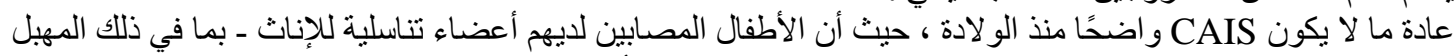

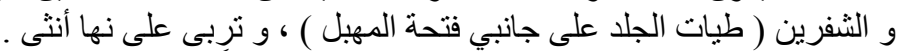

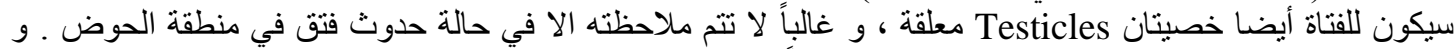

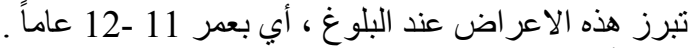

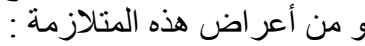

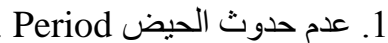
2. نمو القليل من شعر العانة و الأبط. 3. نمو الثديين و حدوث طفر ات نمو سريعة كالمعتاد لكنها تبدو أطول قليلاً من الفتيات بعمر ها .

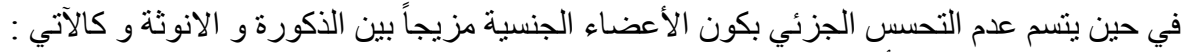

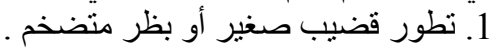
2.

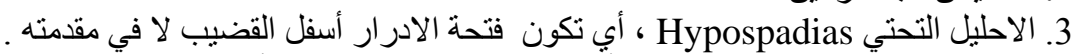

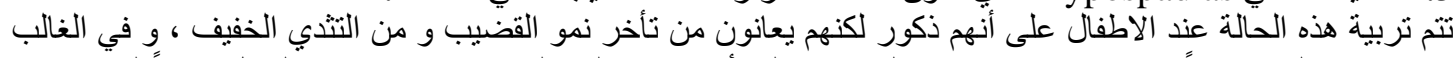

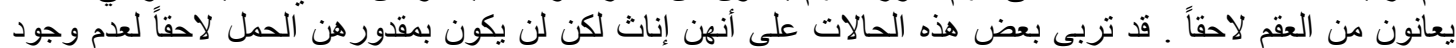

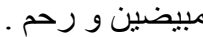

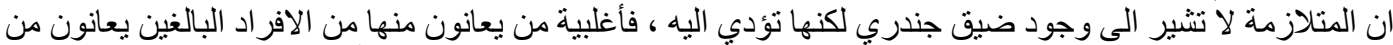

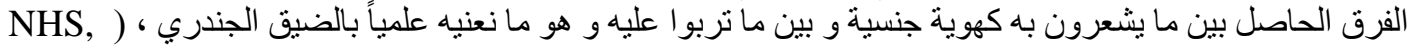
. ( 2018

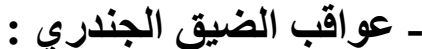

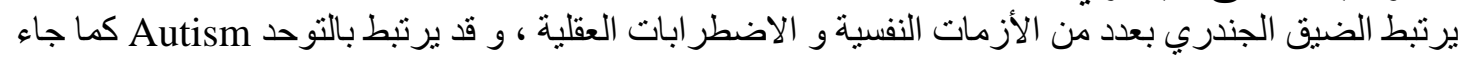

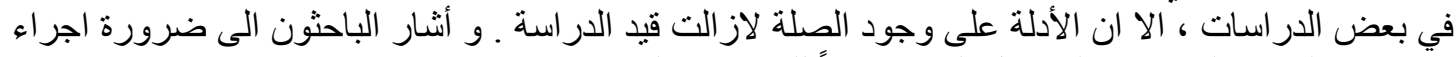

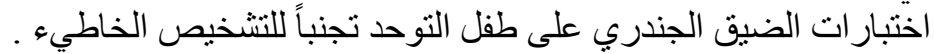

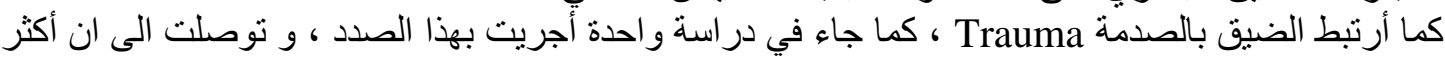

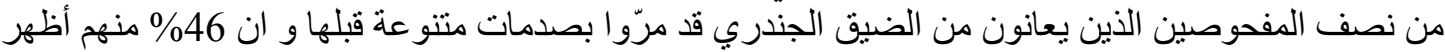

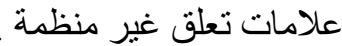
و أرتبط الضيق بالاكتئاب ، إذ ان نصف المفحوصين الذين بعانون من الضيق قد تم تشخيص اصابتهم بالاكتئاب

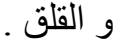
و تزداد نسب الانتحار بين الافراد المصابين بالضيق الجندري بالمقارنة مع عموم السكان ، إذ تبلغ نسبة الإبة

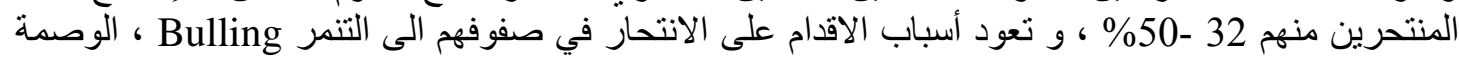

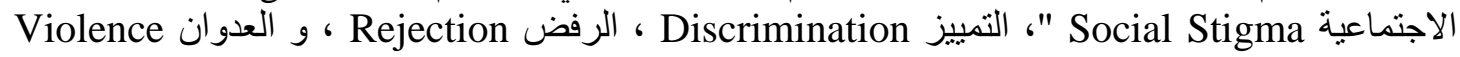

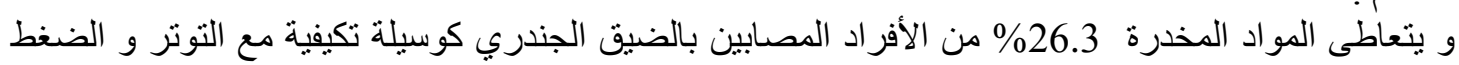

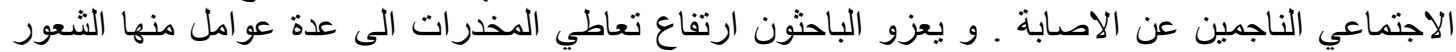

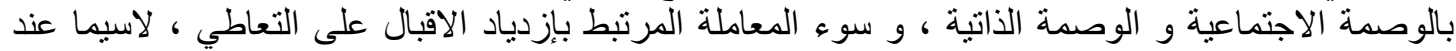

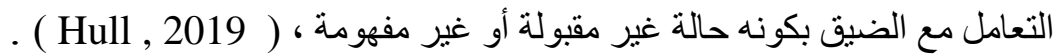

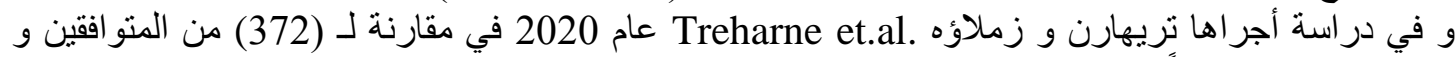

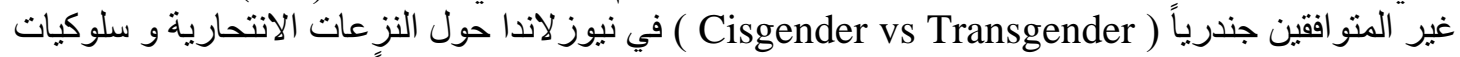
إيذاء الذات ، و توصلت الى ان السلوكيات و النزعات الانتحارية كانت كثر شيو عاً في صفوف غئ الانير المنو افقين 


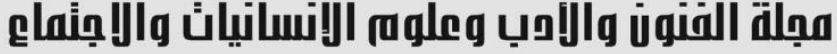

Journal of Arts, Literature, Humanities and Social Sciences www.jalhss.com

\section{Volume (59) October 2020}

العدد (59) اكتوبر 2020

جندرياً و ان الثدة الانفعالية و انخفاض الدعم الاجتماعي برفعان من إحتمالية الاقدام على مثل هذه السلوكيات ،

. ( Treharne et.al. , 2020)

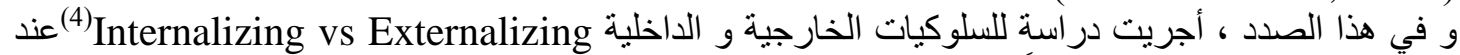

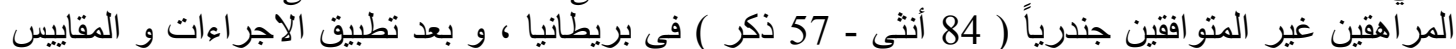

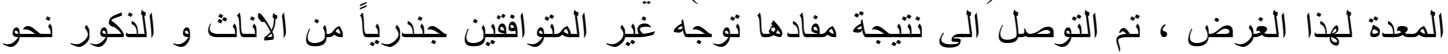

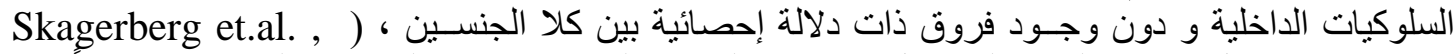
2013 ) ـ و يستدل من هذه النتائج الى ميول نحو ايذاء الذات و التفكير بالانتحار لدى غير المتو الفقين جندرياً.

\section{ـ مُصطلحات ذات صلة بالضيق الجندري :}

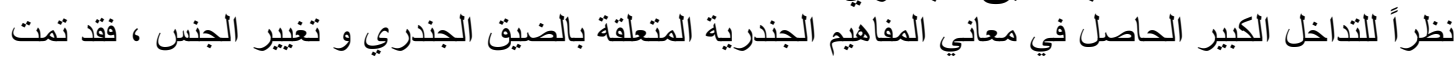

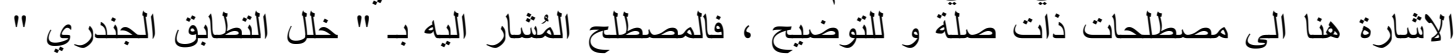
Gender Nonconformity

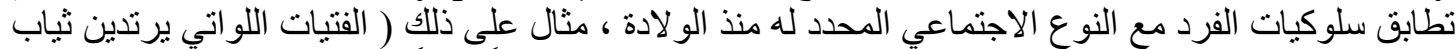

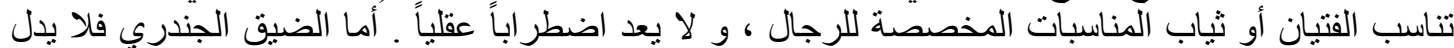

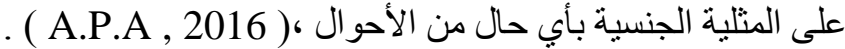

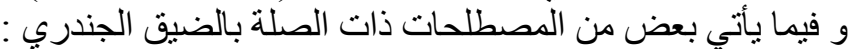

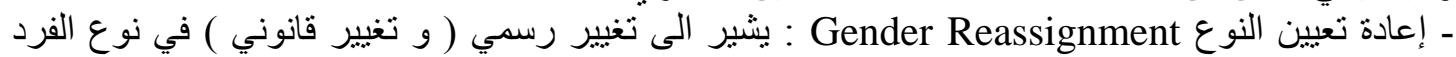

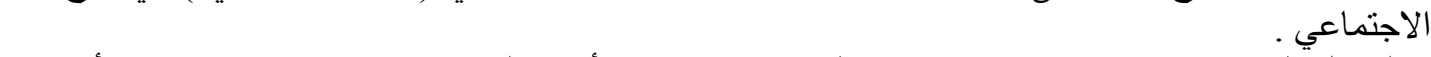
ـ التحول الجندري Transgender : يشير الى مجموعة من الأفراد الذين يتحددون بصورة مستمرة أو عابرة

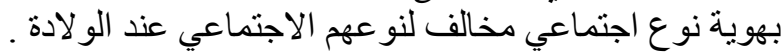

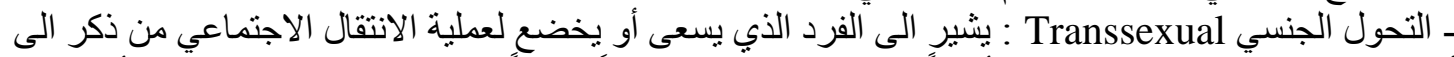

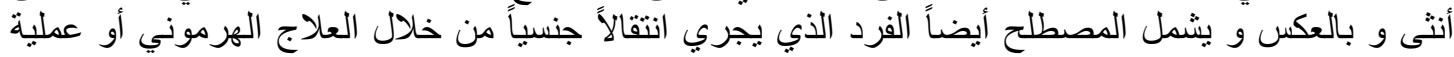

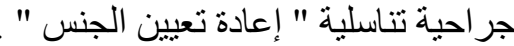
- جندر كوير Genderqueer : أو الهوية الجندرية الحرة ، غير العادئ المحدد: و يشير الى محاولة طمس الهوية الجنسية و التوجه الجنسي من قبل الفرد ـ و عادةً ما يتبنى هؤلاء الافرة اد المرونة في الهوية الجندرية و في لئي التوجه الجنسي .

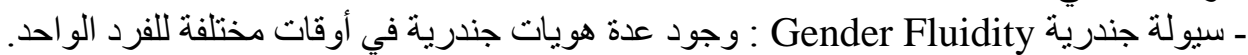

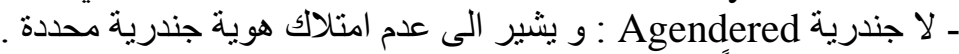
ـ المتو افق جندرياً Cisgender : و و يشير الى الفرد الذي تنطابق هويته الجندرية مع نوعه الاجتماعي عند و لادته. ـ التوسع الجندري Gender Expansiveness : و يشير الى المرونة على صعيد الهوية الجنسية بالمقارنة مع

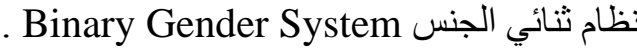
ـ التعبير الجندري Gender Expression : و فيثنير الى الطريقة التي يتو اصل بها الفرد جندرياً مع الآخرين

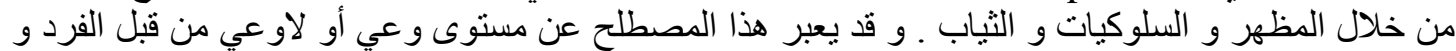

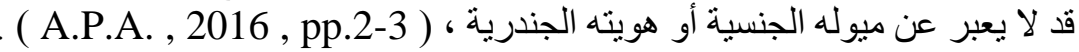

$$
\text { ـ التماتملة : التمل الاستنتاجات و التوصيات الآتية : }
$$




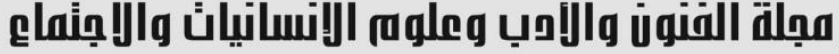

Journal of Arts, Literature, Humanities and Social Sciences www.jalhss.com

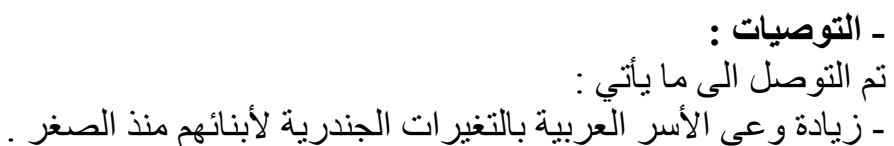

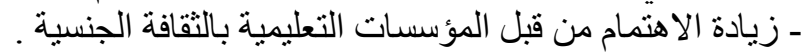

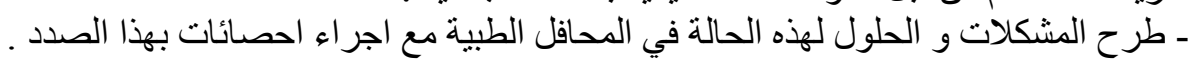

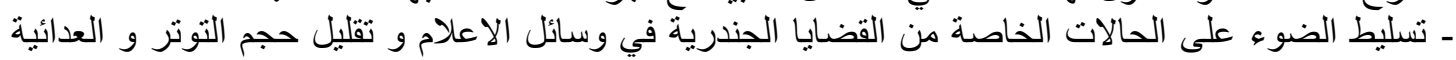
نحو هذه الفئة .

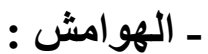

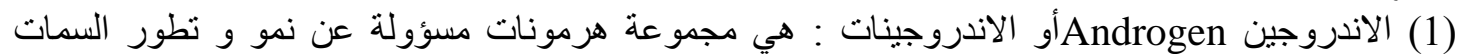

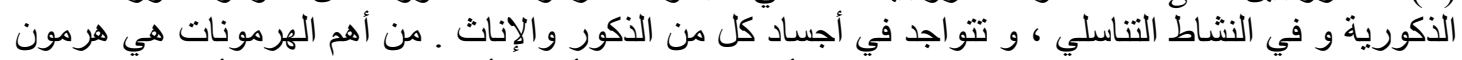

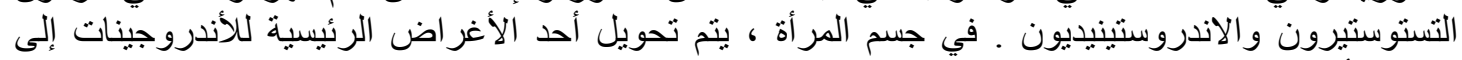

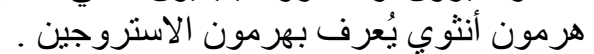
James Simon (n.d.): Androgen, at : https://www.healthywomen.org/yourhealth/androgen

(2) مصطلح أوتوجينيفيليا Autogynephilia: طرحه بلانجارد ، و يشير الى نشاط جنسي متمثل برغبة الفرد

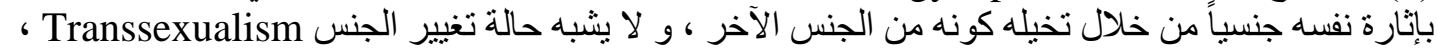
Urban Dictionary (n.d.) : Autogynephilia, at :

https://www.urbandictionary.com/define.php?term=autogynephilia

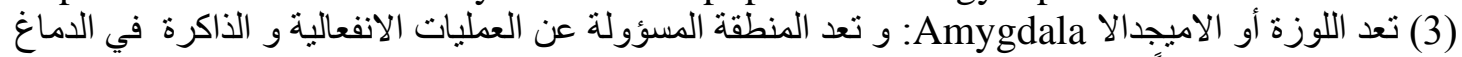

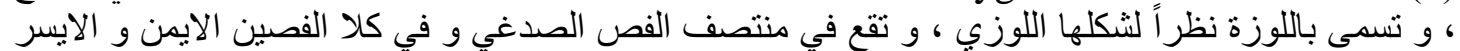

C. Daniel Salzman (n.d.) : Amygdala, Encyclopedia Britannica, at :

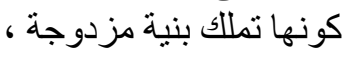
https://www.britannica.com/science/amygdala

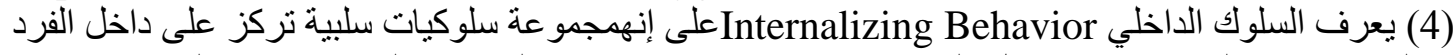

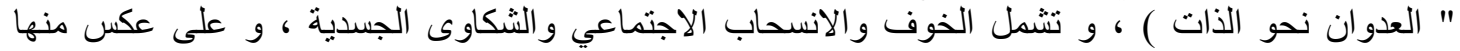

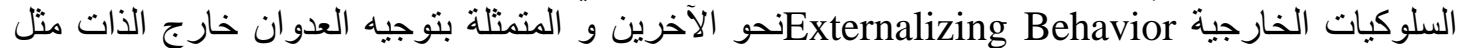
التنمر و التخريب و الحرق العدد تعد أمثلة على السلوكيات الخارجية ،

Yolanda Williams (2015) : Internalizing Behaviors: Definition \& Examples , at : https://study.com/academy/lesson/internalizing-behaviors-definition-examplesquiz.html 


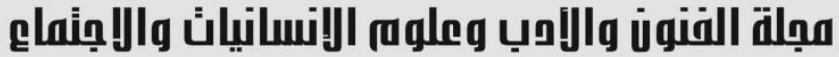 \\ Journal of Arts, Literature, Humanities and Social Sciences www.jalhss.com

\section{References}

1- American Psychiatry Association (2013):Diagnostic and Statistical Manual of Mental Disorders (DSM-5) (5th ed.), Washington, DC and London, American Psychiatric Publishing. pp. 451-460. ISBN 978-0-89042-555-8.

2- American Psychiatry Association (2016) : What is Gender Dysphoria ?, American Psychiatric Publishing,www.psychiatry.org .

3- Blanchard, Ray (1989): The classification and labeling of nonhomosexual gender dysphorias , Archives Of Sexual Behavior, Vol.18 , P.315-334 .

4- Brown , R. George (2019) : Gender Dysphoria and Transsexualism, East Tennessee State University .

5- Canadian Psychological Association CPA (2014) : Psychology Works" Fact Sheet: Gender Dysphoria in Adolescents and Adults ,https://cpa.ca/docs/File/Publications/FactSheets/PsychologyWorksFactSheet_Gen derDysphoriaInAdolescentsAndAdults.pdf

6- Coleman E. et.al. (2011) : Standards of Care for the Health of Transsexual, Transgender, and Gender - Nonconforming People, Version 7 , International Journal of Transgenderism, 13 .

7- Davy, Zowie; Toze, Michael.(2019) :"What Is Gender Dysphoria? A Critical Systematic Narrative Review", Mary Ann Liebert, Inc. Publishers. Mary Ann Liebert, Inc. Publishers.

8- de Jesus,Jaqueline Gomes ; Belden ,C. Micha ; Huynh Hy V. ;Malta ,Monica ; LeGrand ,Sara ; Kaza , Venkata Gopala Krishna \&show all (2020) : Mental health and challenges of transgender women: a qualitative study in Brazil and India , https://doi.org/10.1080/26895269.2020.1761923

9- Garcia-Falgueras A.\& Swaab , D.F.(2010) : Sexual Hormones and the Brain: An Essential Alliance for Sexual Identity and Sexual Orientation, Vol. 17, P. 22-35,

https://doi.org/10.1159/000262525

10- Gerg , Garima ; Elshimy , Ghada ; Marwaha , Raman (2020) :Gender Dysphoria ( Sexual Identity Disorders ), StatPeral Publishing LLC. , Bookshelf ID: NBK532313PMID: 30335346

11- Gliske, Stephen V. (2019) : A New Theory of Gender Dysphoria Incorporating the Distress, Social Behavioral, and Body-Ownership Networks, Cognition and Behavior , eNeuro 2 December 2019, 6 (6) ENEURO.0183-19.2019; DOI: https://doi.org/10.1523/ENEURO.0183-19.2019 .

12- Guillamon, Antonio ; Junque, Carme \& Go'mes - Gil4, Esther (2016) : A Review of the Status of Brain Structure Research in Transsexualism, Arch Sex Behav. , 45, p.1615-1648 , doi: 10.1007/s10508-016-0768-5 .

13- Hare L, Bernard P, Sánchez FJ, Baird PN, Vilain E, Kennedy T \& Harley VR (January 2009): "Androgen receptor repeat length polymorphism associated with male-to-female Transsexualism", Biological Psychiatry , 65 (1) , p. 93-6 , doi:10.1016/j.biopsych.2008.08.033. PMC 3402034. PMID 18962445 


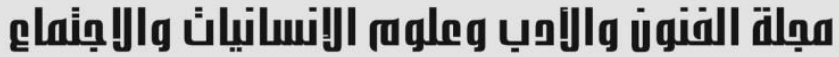 \\ Journal of Arts, Literature, Humanities and Social Sciences www.jalhss.com

14- Heino R. , Kaltiala et.al. (2018) : Gender Dysphoria in Adolescence : Current Perspective, Dove Medical Press Limited, dovepress.com .

15- Hull , Megan (2019) : Gender Dysphoria Statistic, The Recovery Village.

16- Knudson , Gail ; Cuypere , Griet D. ; Bockting , Walter ( 2011) : Second Response of the World Professional Association for Transgender Health to the Proposed Revision of the Diagnosis of Transvestic Disorder for DSM 5 ,P. 9-12 , https://doi.org/10.1080/15532739.2011.606195

17- Lewis , Sophie (2019) : World Health Organization removes "gender identity disorder" from list of mental illnesses, CBS News .

18- Reisner, Sari L. et.al. (2017) : Social Epidemiology for Depression and Anxiety by Gender Identity, Journal of Adolescence Health , 59(2).

19- Saleem , Fatima \& Rizvi , Syed W. (2017) : Transgender Associations and Possible Etiology: A Literature Review, Cureus , 9(12).

20-Skagerberg ,Elin ; Davidson , Sarah \&Carmichael , Polly (2013) :Internalizing and Externalizing Behaviors in a Group of Young People with Gender Dysphoria , P.105-112, https://doi.org/10.1080/15532739.2013.822340 .

21- Treharne, Gareth J. ; Riggs , Damien W. ; Ellis , Sanja J. ; Flett , Jayda A.M. \& Bartholomaeus, Clare (2020) : Suicidality, self-harm, and their correlates among transgender and Cisgender people living in Aotearoa/New Zealand or Australia,

https://doi.org/10.1080/26895269.2020.1795959

22- Vega , Elena - Garcia et.al. (2018) : Suicidal Ideation and Suicide Attempts in persons with Gender Dysphoria ,Psicothema, Vol.30 , No.3.

23- World Health Organization W.H.O.( 2016 ) : International Statistical Classification of Diseases and Related Health Problems 10th Revision (ICD-10)WHO Version for, www.who.int .

24- World Health Organization W.H.O. (2017) : Gender, women and health "What do we mean by "sex" and "gender"?", www.who.int/gender/whatisgender .

25- Zucker , Kenneth J. et.al. (2015) : Gender Dysphoria in Adults , The Annual Review of Clinical Psychology, Vol.11, No.55 . 